eISSN: $2387-1555$

DOI: http://dx.doi.org/10.14201/rea201974153

\title{
PLURIACTIVIDAD, CONSUMO Y PERSISTENCIA DEL MAÍZ EN DOS MUNICIPIOS DEL NOROESTE DEL ESTADO DE MÉXICO ${ }^{1}$ Pluriactivity and Maize Consumption and Persistence in two municipalities of northwestern State of Mexico
}

\section{Estela MARTÍNEZ BORREGO}

Instituto de Investigaciones Sociales, Universidad Nacional Autónoma de México. \mborrego@unam.mx

\section{Janett VALLEJO ROMÁN}

Centro de Investigaciones y Estudios Superiores en Antropología Social - Unidad Golfo.

\jvallejo@ciesas.edu.mx

Fecha de recepción: 28 de octubre de 2018

Fecha de aceptación: 4 de diciembre de 2018

RESUMEN: El objetivo central de este trabajo es describir y analizar la importancia socioeconómica y cultural del cultivo del maíz. A partir de datos obtenidos por medio de entrevistas, cuestionarios y trabajo etnográfico, se busca indagar cómo el maíz, y particularmente el sistema de milpa, ha persistido a pesar de las políticas económicas neoliberales poco favorables para los pequeños productores agrícolas en dos municipios del noroeste del Estado de México: Atlacomulco y San Felipe del Progreso. Algunas de las conclusiones son que la actividad agrícola, a pesar de estar en una continua crisis, permanece como un elemento de identidad y arraigo que responde a factores socioculturales de larga data, como es el consumo del maíz en una gran variedad de formas, así como otros productos asociados a la milpa. Sostenemos que esta persistencia, en términos económicos, es posible dada la presencia de las actividades no agrícolas. En este sentido, la pluriactividad, la reconversión económica y las distintas movilidades se presentan como las principales fuentes para la obtención de ingresos para la sobrevivencia de las familias y del cultivo de maíz.

Palabras clave: agricultura; consumo; pluriactividad; maíz; nueva ruralidad.

ABSTRACT: This paper's main objective is to describe and analyze the socioeconomic and cultural importance of maize production. Based on information obtained through interviews, questionnaires and ethnographic field work, we seek to inquire how maize, and more specifically the milpa system, has persisted despite the negative impacts of neoliberal economic policies on small farmers in two municipalities in the northwest of the State of Mexico: Atlacomulco and San Felipe del Progreso. One of our findings is that, even though agricultural activities have undergone a sustained crisis, they continue to be carried out because they are rooted and are part of people's identity, responding to long-lasting sociocultural factors, such as the consumption of maize in a large variety of forms, as well as the consumption of other products associated with the milpa system. We contend that this persistence is possible in economic terms because of the presence of non-agricultural activities. In this way, pluriactivity, economic reconversion and different forms of mobility have become the main sources of income that allow families to survive and that lead to the persistence of maize production.

Keywords: agriculture; consumption; pluriactivity; maize; new rurality.

RESUMO: O principal objetivo deste trabalho é descrever e analisar a importância socioeconômica e cultural do cultivo do milho. Com base em dados obtidos por meio de entrevistas, questionários e trabalho etnográfico, busca-se investigar como o milho e, particularmente, o sistema da «milpa», tem persistido, apesar das políticas econômicas neoliberais que não favorecem os pequenos produtores agrícolas em dois municípios no noroeste do Estado de México: Atlacomulco e San Felipe del Progreso. Algumas das conclusões apontam que a atividade agrícola, apesar de estar em uma crise contínua, permanece como um elemento de identidade e raízes que responde a fatores socioculturais de longa data, como o consumo de milho em uma grande variedade de formas, assim como outros produtos associados à milpa. Argumentamos que essa persistência, em termos econômicos, é possível graças à presença de atividades não agrícolas. Nesse sentido, a pluriatividade, a reconversão econômica e as diferentes mobilidades são apresentadas como as principais fontes de obtenção de renda para a sobrevivência das famílias e do cultivo do milho.

Palavras chave: agricultura; consumo; pluriatividade; milho; nova ruralidade.

\footnotetext{
${ }^{1}$ Investigación realizada gracias al programa UNAM-PAPIIT IN301417 dentro del proyecto denominado Globalización y procesos de reorganización productiva, social y poblacional en el centro de México adscrito a la Universidad Nacional Autónoma de México.
} 


\section{Introducción}

La inserción de México y sus regiones en la globalización y, más específicamente, en el sistema agroalimentario internacional, ha significado una reconfiguración socioeconómica y espacial regional, en donde los principales rasgos están referidos a la reconversión productiva agrícola dirigida por el Estado bajo un modelo económico neoliberal, que apoya fundamentalmente a los grandes y medianos productores de cultivos comerciales para la exportación, dejando de lado y en la indefensión a la gran mayoría de los productores para el autoconsumo y habitantes del campo.

A partir de ello, asistimos a: un deterioro creciente en las condiciones de existencia de la mayoría de los productores agrícolas y a una crisis de producción y reproducción social; un acelerado proceso de pauperización del espacio; el incremento de empleos informales y precarios o desempleo; la movilidad y la migración de la población, entre otros, que contribuyen al descenso permanente de la capacidad de producción y consumo de bienes y servicios de los productores agrícolas en particular y los pobladores rurales en general. De esta manera, se advierten dos escenarios: 1) intentar integrarse al modelo económico actual y buscar competir bajo parámetros internacionales; o 2) dedicarse a otra(s) actividad(es) para sobrevivir, derivando en una diversificación ocupacional y en la pluriactividad, fenómenos característicos hoy de la realidad en el agro.

Las unidades productivas de una buena parte de los medianos y casi la totalidad de los pequeños productores no aseguran más la reproducción de las familias, por lo que se ven obligados a buscar diferentes «estrategias de reproducción»o «estrategias adaptativas» para hacerse con los recursos necesarios para sobrevivir. Entre ellas, destacan la denominada pluriactividad, a la par que la movilidad laboral y la migración, lo que reconfigura el territorio y el espacio económico-social ya que surgen nuevas actividades y relaciones sociales, enmarcadas en otras formas de organización productiva, residencial, familiar y de vida, así como en nuevos vínculos rural-urbanos. La pluriactividad en los hogares rurales no es un acontecimiento reciente; sin embargo, lo que se puede observar en las sociedades rurales actuales es una mayor diversidad de actividades ocupacionales, así como un incremento en la inserción de los distintos miembros del hogar, sin distinción de género, en el mercado de trabajo formal o informal. Estos procesos se han acentuado a la par de una agudización en el declive de las actividades tradicionales y a la ausencia de un nuevo modelo agrícola y pecuario que permitiera absorber la mano de obra existente en el medio rural.

En este contexto, los miembros de estos hogares han creado y adoptado complejas prácticas de sobrevivencia, que incluyen la mezcla de actividades agrícolas y no agrícolas. Hoy en día, las fuentes de ingresos no agrícolas se han consolidado como el principal sustento de los hogares rurales pero, sobre todo, estas se han sumado a las prácticas que contribuyen a preservar el arraigo con la tierra y con cultivos tradicionales, en este caso, el maíz.

En este artículo nos proponemos analizar la importancia del cultivo del maíz y, en términos generales, del sistema de milpa ${ }^{2}$, como un elemento fundamental para la comprensión del consumo de este grano y en tanto que elementos de arraigo e identidad, sostenidos cada vez más por actividades distintas a las agrícolas y/o pecuarias. De esta manera, las labores asociadas a la pluriactividad, la diversificación ocupacional y a las distintas movilidades son fundamentales para comprender no sólo la permanencia del maíz sino las ruralidades actuales.

\footnotetext{
${ }^{2}$ Milpa viene del náhuatl milli plantación. Se trata de un sistema agrícola tradicional formado por un policultivo que constituye un espacio dinámico de recursos genéticos, más adelante hablaremos más de esto.
} 


\section{Metodología}

La metodología utilizada en esta investigación es de carácter mixto (HERNÁNDEZ et al., 2003; POLE, 2009). Se instrumentó una combinación de técnicas de trabajo de campo para recolectar información cualitativa y cuantitativa, privilegiando el enfoque etnográfico, esto es, la recuperación de prácticas sociales, conocimientos, motivaciones y perspectivas alrededor de la práctica agrícola del maíz y de las distintas ocupaciones que se llevan a cabo para sostener la siembra y garantizar la reproducción del grupo doméstico.

Los resultados de esta investigación son producto de un seguimiento en la zona de estudio desde 2010 y, de manera más permanente, a partir de 2016. Se realizaron varias etapas de trabajo de campo en los municipios de Atlacomulco y San Felipe del Progreso. En 2017 se inició la instrumentación de un cuestionario cerrado, aplicando 137 instrumentos en El Carmen Ocotepec y San Lucas Ocotepec, localidades del municipio de San Felipe del Progreso, y en San Pedro del Rosal y San Antonio Enchisi, en el municipio de Atlacomulco. Este ejercicio permitió la obtención de datos generales acerca de la constitución de los hogares, ocupaciones y presencia de la actividad agrícola, además de registrar la pluriactividad en distintos miembros del hogar; también nos permitió una mayor presencia en las comunidades y lograr realizar 30 entrevistas a profundidad en ambos municipios con milperos y pobladores en general. Como se mencionó, consideramos que esta aproximación teórica metodológica puede ayudar al entendimiento de la situación actual de espacios rurales en donde existen cultivos tradicionales que son importantes en términos socioeconómicos y culturales.

\section{La importancia de la milpa y el maíz}

La siembra, particularmente de maíz, tiene un significado muy importante en términos socioeconómicos y culturales. La importancia del maíz ha trascendido desde los tiempos prehispánicos hasta nuestros días. Los indígenas mesoamericanos expresaron dicha importancia en textos como el Popol Vuh y el códice Chimalpopoca, donde los mitos puntualizaban el carácter esencial de este producto con una doble función: el origen de la existencia humana y el mantenimiento de la misma. Posteriormente, los cronistas de la conquista no ocultaron su asombro sobre la capacidad de transformación y uso de la planta de maíz para diversos fines, pero lo que mayor sorpresa causó, fue la transformación del grano en nixtamal a tortilla, equiparándola con una espacie de pan (TORRES, 2009: 56).

Aunque existe abundante literatura sobre la importancia del maíz (BONFIL, 1984; FERNÁNDEZ, MORALES y GALVEZ, 2012; FLORESCANO, 2016; RABIELA y GUTIERREZ, 2016), aquí destacamos el trabajo de WARMAN (1988) sobre el maíz y el capitalismo. En esta obra se describe cómo fue que este cereal poco conocido fue expandiéndose desde América hacia Europa y África, constituyéndose en una materia prima imprescindible para la industria, la ganadería y la alimentación. El maíz, un cereal «bastardo» por no ser hijo legítimo de las grandes civilizaciones europeas, se integró a los procesos de acumulación del capital. La producción de maíz tenía múltiples ventajas: un cereal «noble» para su cultivo, con altos volúmenes en su cosecha, costos de producción más bajos. Estas ventajas también ayudaron a reducir el costo de alimentación de los esclavos y del ganado.

En la actualidad, este cereal se ha introducido de lleno en el mercado mundial, ya sea por su destino comercial para la alimentación humana y de ganado, o más recientemente para la producción de biocombustible. En México, la relación que existe entre el maíz y el capitalismo se puede observar en el crecimiento que han 
tenido las grandes empresas productoras de harina de maíz ${ }^{3}$ y otros derivados para la alimentación humana. De la misma forma, se ha incrementado la presencia de grandes empresas productoras de semilla transgénica como Monsanto, Syngenta, Dow, AgroScience, Dupont, Bayer CropSciences y BASF, que de acuerdo con GONZÁLEZ y ÁVILA (2014) representan 56\% de la venta de semilla de maíz a nivel mundial. A pesar de la importancia del maíz en la industria y de su inserción en la cadena de producción capitalista, no se ha eliminado su papel primordial en el aspecto culinario y cultural de la población, así como en las expresiones locales y regionales que tiene el maíz para muchos pueblos; y ha sido en los espacios rurales en donde se puede observar cierta contraposición entre la lógica capitalista neoliberal y las prácticas locales y cotidianas de los habitantes.

El objetivo principal de las políticas dirigidas a la producción en masa de cereal ha sido el incremento de los rendimientos, lo que ha sacrificado la calidad desde el punto de vista de los consumidores-productores. De este modo, estas lógicas de producción-consumo se enfrentan con las prácticas de resistencia local. Esta resistencia se materializa en el trabajo continuo y férreo por la conservación de la semilla criolla y en el «saber hacer» de los milperos, cuyo fin último es la conservación de la calidad del producto.

Sin embargo, estas prácticas de resistencia tienen que ver más con una estrategia de sobrevivencia, se enmarcan en una profunda crisis agrícola, consecuencia de la adopción del modelo neoliberal, particularmente dentro de las políticas de liberación comercial y aquellas relacionadas con el desmantelamiento de las instituciones que subsidiaban a los agricultores (APPENDINI, CORTÉS y DÍAZ, 2008; MARTÍNEZ, 1991). De esta manera, en las últimas décadas, los productores de maíz de subsistencia o milperos han tenido que emprender prácticas socioeconómicas que les permitan continuar con la producción, principalmente para el autoconsumo, pues el significado del maíz y de la siembra de la milpa va más allá de un valor económico y es la base fundamental de su consumo.

De acuerdo con LOZADA ARANDA et al. (2017: 10-13), México es el centro de domesticación y diversificación de muchas plantas, proceso que se ha desarrollado en diversos sistemas agrícolas tradicionales. Uno de los más conocidos es la milpa, un agroecosistema que surgió en Mesoamérica y posteriormente se expandió al resto de México y Sudamérica. Se caracteriza por ser un policultivo de especies domesticadas (frijoles, calabazas, chiles, tomates, habas) y otras tantas semidomesticadas (diversidad de quelites, malvas, hongos) los cuales crecen dentro del sistema de forma natural, y el ser humano los ha manejado y protegido con esta modalidad de sembrado.

La siembra de la milpa es parte importante dentro de la alimentación de los pobladores. En este sentido, es significativo insistir que en los distintos estadios de milpa se obtienen otros productos que son igualmente valorados como el maíz. Cuando la milpa es pequeña crecen ahí los quelites, verdolagas, quintoniles, quelites cenizos, malvas, lenguas de vaca, entre otras hierbas silvestres comestibles que se utilizan en la elaboración de diversos platillos.

Además, dentro de la milpa se siembra calabaza, frijol y haba, que se cultivan poco antes de que esté maduro el maíz. En todo este ciclo, están presentes una amplia diversidad de plantas medicinales y un sin número de flores silvestres que adornan los altares y casas. También se organizan fiestas como el día de San

\footnotetext{
${ }^{3}$ Minsa junto con Maseca (Gruma) controlan el 88\% del mercado nacional de la harina de maíz; estas dos empresas han iniciado un proceso de expansión hacia Centroamérica y EUA. A partir de 1992, la compañía Bimbo se adhiere al mercado de la harina de maíz, producción y distribución, la cual también ha incursionado en el mercado estadounidense (TORRES, 2009). Este autor menciona que los sistemas tradicionales han jugado un papel primordial en esta «resistencia», sobre todo, aquellos basados en las tierras de temporal de mediana o baja productividad han sido desplazados de su acceso a subsidios y han perdido su capacidad de ser los abastecedores de maíz para las ciudades. Estos sistemas tradicionales tienen un papel central en la conservación de germoplasma y en la hibridación del maíz criollo.
} 
Isidro Labrador y celebraciones comunitarias en donde se pide por la siembra y agradecen el día de la cosecha.

Esta riqueza biológica y cultural se manifiesta claramente en los dos municipios de estudio. Como mencionamos, a pesar de la crisis por la que atraviesa el agro, la permanencia de la milpa, y concretamente del maíz, permite entender las dinámicas socioeconómicas actuales y el consumo de las poblaciones rurales.

\section{La producción de maíz y su importancia socioeconómica y cultural en Atlacomulco y San Felipe del Progreso}

Los municipios de Atlacomulco y San Felipe del Progreso se ubican en el noroeste del Estado de México. Cuentan con una población de 100.675 y 134.143 respectivamente (INEGI, 2015). En su territorio albergan un número importante de población indígena. En Atlacomulco se registra poco más del 14\% de hablantes mazahuas y $27 \%$ en San Felipe del Progreso; cabe mencionar que este grupo étnico es el más importante en términos cuantitativos para la entidad.

En términos de infraestructura urbana, el municipio de Atlacomulco, históricamente, ha sido favorecido por políticas de urbanización e industrialización: cuenta con un parque industrial, aunque la principal actividad registrada es el comercio. Otro sector importante son los servicios públicos: en este municipio se concentran las oficinas gubernamentales que brindan servicios a los municipios aledaños, siendo así el centro de operaciones regionales en aspectos como agricultura, ganadería, salud, educación y cuestiones ministeriales.

Aunque en cada municipio existen dependencias del gobierno para realizar trámites como, por ejemplo, los relacionados con la actividad agropecuaria, los pobladores se tienen que trasladar a Atlacomulco en donde se concentran las oficinas regionales. Sin embargo, como se mostrará en el texto, a pesar de que la vocación agrícola ha sido desplazada por otras actividades, la permanencia del cultivo y consumo del maíz es una característica importante en la región.

En contraste, San Felipe del Progreso cuenta con infraestructura básica en términos de carreteras, educación y salud, sin presencia significativa de industria. La actividad económica está concentrada en el sector terciario, principalmente el comercio aunque, a diferencia de Atlacomulco, existe una mayor presencia de la actividad agrícola. En términos generales, se puede decir que las labores que desempeña la población son marginales y restringidas en el ámbito geográfico. Se observa una clara persistencia de tareas que se han realizado desde décadas pasadas como el comercio ambulante, el servicio doméstico, el trabajo en la construcción y la labor agrícola; y un surgimiento de una inmensa gama de empleos por cuenta propia e informales relacionados con el comercio y servicios.

En ambos municipios, la pérdida de rentabilidad de la actividad agrícola ha sido el detonante principal para que los pobladores aumenten o busquen otras alternativas no agrícolas para generar ingresos. Como se muestra en el Cuadro 1, el valor real del maíz se ha depreciado gravemente en las últimas décadas. Y como dice un agricultor, «una Coca-Cola vale más que el esfuerzo por lograr un kilo de maíz» (entrevista al Señor Carlos, 2012).

Sin embargo, a pesar de la baja rentabilidad del cultivo del maíz, este continúa sembrándose, sobre todo en San Felipe del Progreso debido a que, quizá por sus raíces prehispánicas. 


\begin{tabular}{|c|c|c|c|c|}
\hline \multirow{2}{*}{ Precio Insumos* } & \multicolumn{4}{|c|}{ Años } \\
\cline { 2 - 5 } & 1982 & 1996 & 2012 & 2017 \\
\hline Litros de gasolina por 1 kg. de maíz & 1.5 & 0.463 & 0.238 & 0.150 \\
\hline Litros de diesel por1 kg. de maíz & 3.5 & 0.582 & 0.248 & 0.140 \\
\hline Kgs. de maíz para comprar fertilizante fórmula 120-60-30 & 194.8 & 626.6 & 592.8 & 480 \\
\hline Kgs. de maíz para pagar el sueldo de un peón & 1 & 20 & 35 & 48 \\
\hline
\end{tabular}

Fig. n. ${ }^{\circ 1}$. Poder de compra del maíz *Precios Junio 2017 Fuente: elaboración propia con base en trabajo de campo y PEMEX. http:// wnw.gas.pemex.com.mx/PGPB/Productos $+y+$ servicios/Gas +licuado/Precios/

Su cultivo tiene un significado simbólico y cultural muy importante y, si bien ya no es la actividad por medio de la cual las familias obtienen los ingresos suficientes para su sobrevivencia, sigue siendo para muchos (ancianos y en menor medida para los jóvenes) una actividad significativa y que no se ha dejado de practicar, como se señala en el siguiente testimonio.

\footnotetext{
El maíz, lejos de decirte que es para autoconsumo, es una costumbre, hay que producir maíz, por ejemplo, mi suegra dice «bay que sembrar porque en tiempos de las elotadas hay que buscar elotes, hay que comer elotes», entonces como que es costumbre, como dicen «si no siembro, ¿qué voy a comer?, necesito comer elotes en mes de julio y agosto, porque comprar elotes siempre es caro». Otra de las cosas que le digo es para autoconsumo porque aquí otra de las cosas es el consumo más fuerte de la gente, que es las tortillas, porque siempre se dice «bueno no tenemos dinero, pero tenemos el maín, suficiente maíz, para hacer tortillas y de comer lo que haiga (sic)", a lo mejor tenemos por hay unos pollitos, unos buevos de casa, pues con eso (Entrevista al Señor Mario, productor, abril de 2017).
}

Además, la producción de maíz constituye también un aporte en especie muy importante para la sobrevivencia de las familias. El ahorro económico que genera su cultivo se materializa principalmente en el consumo de tortilla y de otros productos secundarios como el atole, tamales o pinole y, en menor medida, se utiliza en la alimentación de algunos animales de traspatio. Por otra parte, la búsqueda de calidad en el consumo de la tortilla continúa siendo fundamental en esta región (APPENDINI y QUIJADA, 2015). El cultivo de maíz en sus variedades criollas ha sido una práctica de los productores rurales mexicanos, quienes obtienen maíz de calidad de acuerdo con sus preferencias y cultura; más allá de los mercados en donde es posible adquirir los alimentos básicos derivados del maíz a precios que a veces resultan más bajos que la producción propia. Esto se refleja en los siguientes testimonios:

\footnotetext{
El maíz es parte de la alimentación de la familia, habiendo maíz, aunque no haya comida, con eso nosotros estamos muy satisfechos...Pues no [lo dejaría de cultivar] porque es parte de nuestra alimentación..un ejemplo, el maíz es barato, pero también ya convertirlo en tortilla es caro, y entonces para nosotros jamprar tortillas diario?...., un ejemplo, dependiendo de la familia, que seas unos 5 ó 6, ya tres kilos diarios y tres de comida son como 80 pesos, ya genera un gasto, y ya tener el maí, ya no, porque nosotros lo preparamos. (Entrevista al Señor Vicente, productor, abril de 2017).
}

Pues sí, yo pienso que puede salir más barato comprar la tortilla o ya por lo menos te evitas la friega, menos trabajo. Pero muchas veces a la gente no le gusta la tortilla de la tortillería; entonces yo pienso que siguen echando [elaborando] tortilla porque, vamos, ya lo traen de arraigo, de muy atrás, pero más que nada, la situación de la calidad de la tortilla, es más nutritiva. (Entrevista al Señor José, febrero de 2010).

Las de la tortillería son muy delgaditas; cuando uno las va a calentar se ponen tiesas, parecen suelas. La tortilla que nosotros hacemos en la casa, en primera, es más gruesa, y cuando usted la calienta queda blandita, blandita. (Entrevista al Señor Benito, mayo de 2012).

Esta permanencia de prácticas como el consumo de tortilla vinculada fuertemente a la producción de maíz, se ve reflejada en la superficie sembrada de maíz. A pesar de que en ambos municipios ha tenido variaciones importantes al alza o a la baja de 1970 y hasta 2015 (cuadros 2 y 3), es importante notar que a partir del 
2002 la superficie se mantiene con variaciones poco significativas.

\begin{tabular}{|c|c|c|c|c|c|c|c|c|c|}
\hline \multirow{2}{*}{ MUNICIPIO } & \multicolumn{10}{|c|}{ AÑOS } \\
\cline { 2 - 10 } & 1970 & 1991 & 2002 & 2010 & 2011 & 2012 & 2013 & 2014 & 2015 \\
\hline Atlacomulco & 3,457 & 7,275 & 12,863 & 11,800 & 11,720 & 12,257 & 12,225 & 12,195 & 12,162 \\
\hline San Felipe del Progreso & 17,899 & 40,761 & 25,150 & 23,890 & 21,000 & 23,500 & 23,232 & 23,293 & 23,308 \\
\hline
\end{tabular}

Fig. n. ${ }^{\circ}$. Superficie sembrada de maíz (ha) en los municipios de Atlacomulco y San Felipe del Progreso 1970-2015.

Fuente: Elaboración propia con datos del V y VII Censos Agrícola-Ganadero, 1970 y 1994.

Oficina Estatal de Información para el Desarrollo Sustentable del Estado de México (OIEDRUS-Estado de México). Disponible en http://www.campomexiquense.gob.mx/Cuadro

\begin{tabular}{|c|c|c|c|c|c|c|c|c|}
\hline MUNICIPIO & $\begin{array}{c}\text { Cambio } \\
1970-1991\end{array}$ & $\%$ & $\begin{array}{c}\text { Cambio } \\
1991-2002\end{array}$ & $\%$ & $\begin{array}{c}\text { Cambio } \\
2002-2010\end{array}$ & $\%$ & $\begin{array}{c}\text { Cambio } \\
2010- \\
2015\end{array}$ & $\%$ \\
\hline Atlacomulco & $3,817.63$ & 110.43 & $5,588.27$ & 76.82 & $-1,063.00$ & -8.26 & 2,308 & 19.55 \\
\hline $\begin{array}{c}\text { San Felipe del } \\
\text { Progreso }\end{array}$ & $22,862.25$ & 127.73 & $-15,610.75$ & -38.30 & $-1,260.00$ & -5.01 & -582 & -2.43 \\
\hline
\end{tabular}

$$
\text { Fig. n. }{ }^{\circ} \text {. }
$$

Cambio en la superficie sembrada de maíz (ha) en los municipios de Atlacomulco y San Felipe del Progreso1970-2015.

Fuente: Elaboración propia con datos del V y VII Censos Agrícola-Ganadero, (1970 y 1994) y de la Oficina Estatal de Información para el Desarrollo Sustentable del Estado de México (OIEDRUS-Estado de México).

Disponible en http://www.campomexiquense.gob.mx/Cuadro

La persistencia del cultivo de maíz es posible principalmente por dos factores: 1) el que distintos miembros de la unidad doméstica continúan invirtiendo con trabajo en las labores agrícolas (DÍAZ, 2008), y 2) la transferencia vía ingresos de actividades no agrícolas a la parcela (DÍAZ, 2008). Tal como lo muestran los siguientes testimonios:

\footnotetext{
Mi papá ayuda a una de mis tías en la cosecha porque no puede pagar peones. Ella tiene dos hijos con sus nueras y nietos. Primero se levanta la cosecha de mi papá y luego vamos todos a su terreno... luego yo ya quiero regresar al trabajo, y me dice papá «no, abora hay que ir con tu madrina, que ya es viuda y sus hijos están en el norte»... Entonces otra vez. todos al terreno de la madrina. Ya vengo regresando al mes aqui, a la fábrica. (Entrevista a la Señora Elena, obrera, febrero de 2010).
}

La mayoría aqui cultivamos porque todos comemos la tortilla. Sale caro cultivar, le inviertes, porque como yo, soy mamá soltera, entonces no tengo quien me ayude; tendría que contratar un peón que abora cobra \$80 más su comida, su cerveza (...) ya te sale como en \$100, más el abono, y el líquido. Si le echas lápi:, isale caro! Entonces lo que hace uno es ayudarle a tu familia, hermanos, primos, y cuando te toca sembrar o cosechar a ti, pues ellos te ayudan; es como si les pagaras con trabajo (Entrevista a la Señora María Dolores, mayo de 2010).

Así, la pluriactividad y la diversificación ocupacional (ARIAS, 2009; CARTON DE GRAMMONT, 2008; GRAS, 2004; JARQUÍN et al., 2017; SALAS, 2016) han sido una de las prácticas para sostener la siembra y consumo del maíz. De esta manera, la producción de subsistencia depende cada vez más de los ingresos provenientes de actividades distintas a la agrícola, ya que como el ciclo de cultivo del maíz sólo dura seis meses (ciclo primavera-verano), el resto del año los productores se dedican a otras actividades remuneradas. En trabajo de campo se observó con alta frecuencia casos en los que algún o algunos miembros de la familia aportaban trabajo o dinero para invertir en alguna etapa del cultivo del maíz.

Buena parte de las familias de la región ha tenido que buscar otras actividades fuera de la parcela: el comercio formal e informal muchas veces itinerante, la maquila de confección, la construcción, los servicios, 
etc. Las más de las veces estas actividades implican aceptar trabajos precarios y mal pagados, así como desplazamientos geográficos. Lo anterior queda evidenciado en cierta medida en el Cuadro 4, que nos muestra los cambios en la PEA en los últimos casi 50 años, y en donde podemos ver que la población dedicada exclusivamente al sector primario ha ido disminuyendo a través de las diferentes décadas, lo que no significa que se abandone la agricultura de manera completa.

\begin{tabular}{|c|c|c|c|c|c|}
\hline Municipio & & 1990 & 2000 & 2010 & 2015 \\
\hline \multirow{4}{*}{ Atlacomulco } & PEA & 26.64 & 30.00 & 37.17 & 48.81 \\
\cline { 2 - 6 } & Sector primario \% & 22.35 & 15.46 & 12.50 & 10.35 \\
\cline { 2 - 6 } & Sector secundario \% & 24.35 & 24.67 & 21.14 & 23.57 \\
\cline { 2 - 6 } & Sector terciario \% & 48.53 & 56.39 & 65.59 & 65.28 \\
\hline \multirow{3}{*}{ San Felipe del Progreso } & PEA \% & 22.58 & 21.02 & 32.02 & 40.79 \\
\cline { 2 - 6 } & Sector primario \% & 48.87 & 32.31 & $31.83^{*}$ & 15.36 \\
\cline { 2 - 6 } & Sector secundario \% & 26.35 & 32.51 & $32.53^{*}$ & 39.14 \\
\cline { 2 - 6 } & Sector terciario \% & 18.71 & 31.17 & $35.16^{*}$ & 44.57 \\
\hline
\end{tabular}

Fig. n. ${ }^{\circ}$ 4. Población Económicamente Activa (PEA) según sector de actividad para Atlacomulco y San Felipe del Progreso, 1990, 2000, 2010 y 2015

*Los porcentajes se calcularon a partir de la población ocupada y no de la PEA, debido a la presentación de la información en INEGI para dicho año. Fuente: Elaborado por Lissette Rosales, XI Censo General de Población y Vivienda, INEGI, 1990; XII Censo General de Población y Vivienda, INEGI, 2000; Censo General de Población y Vivienda, INEGI, 2010; y la Encuesta Intercensal 2015.

Destaca el caso de Atlacomulco en donde el sector primario disminuyó alrededor de 50 puntos porcentuales. Esto se explica en buna parte porque, como ya mencionamos, este municipio ha presentado un mayor proceso de urbanización en la región. Y, por el contrario, San Felipe mantiene aún su agricultura muy arraigada, lo que se evidencia en que, para 2010, no existe un sector predominante, los tres sectores representan cifras entre el 30\% y 35\%. No obstante, llama la atención que para el 2105 se presente una reducción de casi el 50\% de la población dedicada a la agricultura. Esto se debe a que, si bien el indicador de la PEA puede ser útil para entender la dinámica laboral grosso modo, este dato no permite tener registro de aquellas personas que son pluriactivas. Esto se corroboró en la aplicación de nuestro instrumento, que a diferencia del registro que ofrece el INEGI, nos permitió registrar pluriactividad, es decir, el registro de otras actividades (remuneradas o no) que realizan las personas durante el año que son fundamentales para la dinámica y reproducción familiar, por ejemplo, mujeres dedicadas a las labores domésticas en su propio hogar, estudiantes que «ayudan» a las labores de campo y personas que con un empleo regular reportan como segunda o tercer actividad las labores agrícolas.

A partir de la aplicación del cuestionario en campo, fue posible identificar un total de 424 personas que cuentan con alguna ocupación productiva, en la cuales fue incluido el trabajo en el hogar, reportado principalmente por las mujeres. Los resultados muestran que 84 personas reportaron el campo como principal ocupación, lo que representa el 20\% del total. En lo que se refiere al campo como actividad secundaria, los datos obtenidos señalan que la actividad agrícola fue reportada como segunda ocupación por un total de 128 personas (37 en Atlacomulco y 91 en San Felipe del Progreso), y por 2 personas como tercera ocupación (1 en cada municipio). Estos datos resultan significativos, pues ya sea como actividad principal o secundaria, el campo es reportado por un total de 214 personas, lo que representa el 50\% del total de personas con actividades productivas. En cuanto a la posición en la estructura familiar, el campo fue reportado entre jefes/as, cónyuges e hijos/as, lo que da cuenta de la importancia del trabajo familiar para el mantenimiento de esta actividad.

De esta forma, el cultivo de maíz se ha vuelto una tarea secundaria, un trabajo de segundo tiempo, o hasta una actividad de «ocio» que se ejerce cuando se puede, sin que por ello, insistimos, no sea fundamental para la reproducción de la familia. No obstante, la PEA sólo registra la actividad principal por individuo en el 
momento de la encuesta y de acuerdo al criterio de la persona. Si sólo consideramos este dato, pudiera pensarse en el «abandono» de la actividad agrícola, lo que contrasta con la realidad, ya que, como se puede constatar in situ, una buena parte de la población continúa sembrando, y lo que vemos es, sobre todo en San Felipe, un paisaje en donde predominan parcelas sembradas con maíz, en su mayoría de temporal, y muy pocos terrenos abandonados.

\footnotetext{
Mi hija me dice que ya no siembre, pero ¿cómo no? si de abi sale para la tortilla, ya pues puedes comer una tortilla de tu maír. Esta vez me costó mucho, no tenía para el abono y la milpita ya jiloteaba, y yo sin dinero [...] pues ya mi nuera me vendió unas servilletas bordadas en Toluca, ya tuve para mi abono. No es mucha mi milpita, es como un cuarto de hectárea, pero bay que comprar el abono, contratar peón, es gasto, es dinero. Hay que buscarle para que sigamos sembrando, ni modo que dejes la tierra al abandono. A mi me la dejo mi difunto esposo y a él se la dio su papá, ni modo que la abandone, le digo a mi bija, hay que trabajar para sembrar. (Entrevista a la Señora María, productora, mayo 2017).
}

Para complementar ingresos y no abandonar por completo la producción del maíz, los milperos de Atlacomulco han iniciado hace aproximadamente una década la producción de productos comerciales como los nopales y diferentes especies de flores. La superficie sembrada del primero pasó de 15 hectáreas en 2003 a 121 en 2015 y los rendimientos de 2.5 a 82 toneladas por hectárea, y el geranio flor de 7 a 17 hectáreas, aunque mantuvo más o menos igual su rendimiento alrededor de las 300 mil toneladas por hectárea en esos mismos años (Cuadro 5).

Por su parte, en San Felipe iniciaron en 2008 el cultivo de jitomate en invernadero, pasando de 65 toneladas a 680 (Cuadro 6), además de que, en ambos, hoy se ponen a la venta productos que anteriormente eran sólo para el autoconsumo: acelgas, cilantro, quelites, y otros, aunque en tan pequeños volúmenes que no aparecen en los registros estadísticos institucionales, pero en las entrevistas sí quedó plasmada su importancia para complementar ingreso.

\begin{tabular}{|c|c|c|c|c|c|c|c|c|c|c|c|c|c|}
\hline Producto & 2003 & 2004 & 2005 & 2006 & 2007 & 2008 & 2009 & 2010 & 2011 & 2012 & 2013 & 2014 & 2015 \\
\hline Máiz grano & 39,749 & 40,220 & 19,013 & 35,314 & 46,908 & 46,130 & 25,247 & 32,892 & 8,220 & 35,601 & 54,533 & 61,482 & 59,336 \\
\hline Máiz forrajero & 22,300 & 22,820 & 20,150 & 28,350 & 31,665 & 30,580 & 22,740 & 26,884 & 21,496 & 20,265 & 41,490 & 42,199 & 39,401 \\
\hline Nopalitos & $22,300.00$ & 22,820 & 20,150 & 28,350 & 31,665 & 30,580 & 22,740 & 26,884 & 21,496 & 20,265 & 41,490 & 42,199 & 39,401 \\
\hline Geranio & 37,5 & 150 & 65 & 84 & 1,280 & 2,250 & 2,340 & 3,584 & 3,648 & 10,146 & $10,100.40$ & 10,032 & 9,590 \\
\hline Rosa & $2,250,000$ & $3,240,000$ & $3,240,100$ & $3,240,000$ & $3,450,000$ & $4,983,000$ & 360,000 & $6,626,300$ & $6,840,000$ & $5,564,160$ & $5,431,680$ & $4,073,969$ & $5,564,311.2$ \\
\hline Noche buena & 960,000 & 9600,000 & $1,200,000$ & $1,440,000$ & $1,398,000$ & $1,594,600$ & 240,000 & $2,042,975$ & $2,400,000$ & $2,160,000$ & $2,203,200$ & $1,555,430$ & $2,203,455$ \\
\hline
\end{tabular}

Fig. n. ${ }^{\circ}$. Atlacomulco, producción por cultivos básicos y perenes (tons.) 2003-2015

Fuente: elaboración propia con datos tomados del Sistema de Información Agropecuaria y Pesquera.

Disponible en: http://infosiap.siap.gob.mx/aagricola_siap_gb/cultivo/index.jsp

\begin{tabular}{|c|c|c|c|c|c|c|c|c|c|c|c|c|c|}
\hline Producto & 2003 & 2004 & 2005 & 2006 & 2007 & 2008 & 2009 & 2010 & 2011 & 2012 & 2013 & 2014 & 2015 \\
\hline Maiz grano & $59,922.9$ & $72,973.4$ & $58,046.9$ & $61,779.3$ & $77,471.50$ & $74,431.8$ & $50,036.0$ & $63,929.8$ & $12,035.0$ & $58,014.0$ & $87,247.5$ & $75,674.9$ & $88,616.10$ \\
\hline Papa & $3,000.0$ & $1,225.0$ & $1,064.0$ & $1,400.00$ & $1,050.00$ & 750.00 & 840.00 & $1,440.0$ & $1,8000.0$ & $2,340.0$ & $2,528.4$ & $2,250.0$ & $1,506.00$ \\
\hline Tomate verde & 450.00 & 595.0 & 270.0 & 280.00 & 400.00 & 774.00 & 562.50 & 702.0 & 950.0 & 918.00 & 865.0 & 774.0 & 700.00 \\
\hline $\begin{array}{c}\text { Tomate roj0 } \\
\text { (jitomate) }\end{array}$ & 0 & 0 & 0 & 0 & 0 & 65.00 & 160.0 & 420.00 & 350.00 & 444.0 & 475.0 & 651.0 & 680.00 \\
\hline
\end{tabular}

Fig. n. ${ }^{\circ}$ 6. San Felipe del Progreso, producción producción por cultivos básicos y perenes (tons.) 2003-2015 Fuente: elaboración propia con datos tomados del Sistema de Información Agropecuaria y Pesquera. Disponible en: http://infosiap.siap.gob.mx/aagricola_siap_gb/cultivo/index.js 
En este contexto, la pluriactividad se consolida como una de las características actuales de los espacios rurales. Los aspectos que favorecen la pluriactividad son diversos. Se pueden mencionar aquellos elementos que influyen en las transformaciones territoriales y económicas, entre las cuales destaca el cambio en la relación rural-urbana como consecuencia de la reestructuración laboral y relocalización de actividades favorecida por la cercanía con las ciudades, el incremento de vías de comunicación y medios de transporte, que propician una movilidad laboral. De esta manera, en la mayor parte de los casos, el fenómeno de la «pluriactividad» campesina no puede ser separado de la movilidad geográfica, ya que los empleos alternativos se ubican generalmente fuera de los espacios agrícolas y, en muchos casos, en localidades más alejadas y urbanas.

Otra de las fuentes para adquirir ingresos es la migración temporal o permanente hacia otras partes del país o del extranjero. La aplicación del cuestionario en campo corrobora la importancia de la movilidad para la obtención de ingresos monetarios que permitan la reproducción de los hogares rurales. Tomando en consideración la principal actividad reportada, y excluyendo el trabajo en el hogar y en el campo, 210 personas reportan actividades que pueden generar ingresos monetarios, de las cuales el 59\% (123 personas) tienen que desplazarse fuera de su localidad. Los datos obtenidos señalan que, del total de personas que se desplazan fuera de su localidad, el 25\% va hacia la cabecera municipal; 15\% hacia otro municipio del Estado de México; 24\% hacia la Ciudad de México; 25\% hacia otro estado de la República Mexicana; y 10\% hacia Estados Unidos. Cabe señalar que los destinos migratorios en el país son diversos, pues incluyen entidades colindantes con la región como Michoacán y Querétaro, así como estados más alejados, como Coahuila, Sonora, Puebla o Veracruz, en donde se desarrollan principalmente actividades de albañilería, empleo doméstico, comercio a pequeña escala de diversos productos, y ayudantes en general.

\section{Conclusiones}

Una de las ideas que guiaron la elaboración de este trabajo fue el estudio de la importancia socioeconómica y cultural del cultivo y del consumo del maíz en particular y del sistema de milpa en general entre los habitantes de la región noroeste del Estado de México. Si bien lo «rural» ha trascendido hoy en día lo propiamente agrícola, sostenemos que, en no pocas regiones, y entre ellas la que aquí estudiamos, la actividad agrícola de cultivos tradicionales persiste por cuestiones económicas y también por motivos culturales, destacando lo que corresponde al consumo humano en diferentes vertientes.

En el caso de la región de estudio, el cultivo de maíz asociado a la milpa es parte importante de la alimentación y de distintos aspectos relacionados con la producción y con la preservación de la salud, ya que en sus distintos estadios se obtienen otros productos que son igualmente valorados como los elotes o mazorcas, y distintos tipos de quelites y leguminosas, que están incorporados también en el uso y consumo cotidiano de la población. De esta manera, consideramos que poner en la mesa de discusión la pertinencia de esta práctica agrícola es vigente y necesaria en tanto que sigue constituyéndose como una actividad que proporciona insumos para el consumo y la sobrevivencia de las familias rurales, además de persistir como el agroecosistema de mayor arraigo, extendido y diverso a lo largo del país.

Esta práctica campesina e indígena está fuertemente relacionada con hacer un uso maximizado del recurso, en este caso, el maíz. Algunos de los habitantes manifestaron dejar algo de rastrojo en la tierra para protegerla de la erosión y utilizarlo como un abono natural, lo que ha permitido generar resistencia ante las condiciones climáticas adversas y permite el aumento de nutrientes para el suelo. Por otra parte, dicho producto es también importante en la cría de animales de traspatio (gallinas, guajolotes y patos), ganado menor (cerdos, 
borregos y cabras), y en algunos casos, incluso de ganado mayor (vacas, becerros, mulas, caballos y burros). Todos ellos consumen el maíz de menor calidad y el rastrojo.

Aunado al uso de los recursos materiales generados, otro elemento que ayuda a entender la persistencia del cultivo del maíz es su papel como resguardo económico, aun cuando es un cultivo que ha perdido su valor comercial. Frente a escenarios como enfermedades, accidentes, muerte de algún familiar o gastos familiares inesperados, se recurre a la venta del maíz con los intermediarios, establecidos de manera permanente en bodegas de acaparamiento principalmente en Atlacomulco.

De este manera, se puede señalar que la continuidad del cultivo del maíz tiene varias aristas: a) el ahorro económico que se materializa en el consumo de tortilla y de otros alimentos secundarios como el atole, tamales o pinole; b) como alimento para los animales de traspatio; c) el cultivo del maíz como elemento nodal del sistema de la milpa, el cual ofrece otros productos de consumo alimenticio como el frijol, calabaza, flor de calabaza, diversos tipos de quelites; hongo de milpa; d) como elemento de construcción de identidad en donde se vincula la producción del maíz con la posesión de la tierra; y e) en tiempos de crisis económica familiar el maíz es puesto a la venta.

No obstante, a pesar de las ventajas que ofrece el cultivo del maíz, es incuestionable que en la actualidad las familias rurales dependen de otros trabajos y actividades para adquirir los satisfactores que requieren para su reproducción. Los precios bajos del maíz y el aumento en los costos de los insumos vuelven económicamente insuficiente su cultivo para la manutención familiar, por lo que la alternativa viene a ser el ejercicio de múltiples actividades remuneradas en los hogares rurales.

La pluriactividad ayuda a explicar la persistencia del maíz en un contexto de pauperización de la población rural, pero también es indicativo de las transformaciones espaciales. Las actividades no agrícolas juegan un papel fundamental para la subsistencia de las familias rurales y para satisfacer nuevas necesidades de consumo, principalmente en el sector juvenil, aunque no es exclusivo de este grupo etario. Se observó que el deseo de acceso a tecnologías de comunicación, nueva vestimenta, otras formas de diversión e incluso la incorporación de otros alimentos en la dieta familiar, como la comida rápida y/o procesada, es una constante. Entonces, lo que resulta interesante destacar es que el arraigo con la tierra y el cultivo continúa, a pesar de la presencia de una dinámica poblacional inserta en una lógica de mercado local-global.

Finalmente, es importante hacer mención de la diversidad de casos de productores que encontramos ya que, aunque en su gran mayoría son minifundistas, no todos cuentan con las mismas redes sociales ni los recursos que les faciliten el «ser pluriactivos». Lo que se observó es que quienes son más pluriactivos tienen mayor posibilidad de mantener la producción maicera, por la transferencia vía ingresos para subsidiarla. También se encontró que quienes conservan y ejercen lazos de parentesco extendidos, reducen costos de producción, ya que la ayuda entre familias para las tareas más fuertes, como siembra y cosecha, amortiguan los gastos. No obstante, lo que es invariable es la importancia que sigue teniendo la milpa como elemento indiscutible en la reproducción socioeconómica de las familias.

\section{REFERENCIAS BIBLIOGRÁFICAS}

APPENDINI, K. y QUIJADA, M. G. (2015). «Consumption strategies in Mexican rural households: pursuing food security with quality». Agriculture and Human Values, 32(2), 439-454.

APPENDINI, K., CORTÉS, L. y DÍAZ, J. V. «Estrategias de seguridad alimentaria en los hogares campesinos: la importancia de la calidad del maíz y la tortilla». En APPENDINI, K. y TORRES-MAZUERA, G. 
(eds.). (2008) ¿Ruralidad sin agricultura?. Ciudad de México: El Colegio de México, pp. 103-128

ARIAS, P. «Nueva estructura Ocupacional en el campo Latinoamericano». En CARTON DE GRAMMONT,

H. y MARTÍNEZ, L. (coord.). (2009) La pluriactividad en el campo latinoamericano. Quito: FLACSO, pp. 171-206.

BONFIL, G. (1984). El maíz fundamento de la cultura popular mexicana. Ciudad de México: Museo Nacional de Culturas Populares, Dirección General de Culturas Populares, SEP.

CARTON DE GRAMMONT, H. «El concepto de nueva ruralidad». En PÉREZ, E., FARAH, M. y CARTON DE GRAMMONT, H. (eds.). (2008) La nueva ruralidad en América Latina. Bogotá: FLACSO, Pontificia Universidad Javeriana, pp. 23-44.

DÍAZ HINOJOSA, J. V. «Valor económico de la seguridad alimentaria de maíz en unidades domésticas productoras del Centro de México». En APPENDINI, K. y TORRES-MAZUERA, G. (eds.). (2008) ¿Ruralidad sin agricultura?. Ciudad de México: El Colegio de México..

FERNÁNDEZ SUAREZ, R., MORALES CHAVEZ, L. A. y GALVEZ MARISCAL, A. (2013). «Importancia de los maíces nativos de México en la dieta nacional: Una revisión indispensable». Rev. fitotec. Mex., 36, 275-283.

FLORESCANO, E. (2016). ¿Cómo se hace un Dios?: creación y recreación de los Dioses en Mesoamérica. Barcelona: Taurus.

GONZÁLEZ, A. y ÁVILA, J. F. (2014). «El maíz en Estados Unidos y en México: Hegemonía en la producción de un cultivo». Argumentos, 27, 215-237.

GRAS, C. (2004). «Pluriactividad en el campo argentino: el caso de los productores del sur santafecino». Cuadernos de Desarrollo Rural, 51, 91-114.

HERNÁNDEZ, R., FERNÁNDEZ, C. y BAPTISTA, M. P. (2003). Metodología de la Investigación. Ciudad de México: Mc. Graw Hill.

INSTITUTO NACIONAL DE ESTADÍSTICA Y GEOGRAFÍA (1970). V Censo Agrícola-Ganadero. Resultados Definitivos. Aguascalientes: INEGI.

INSTITUTO NACIONAL DE ESTADÍSTICA Y GEOGRAFÍA (1990). XI Censos General de Población y Vivienda 1990. [En línea] http://www.beta.inegi.org.mx/proyectos/ccpv/1990/

INSTITUTO NACIONAL DE ESTADÍSTICA Y GEOGRAFÍA (1994). VI Censo Agrícola-Ganadero. Resultados Definitivos. Aguascalientes: INEGI.

INSTITUTO NACIONAL DE ESTADÍSTICA Y GEOGRAFÍA (2000). XII Censos General de Población y Vivienda 2000. [En línea] http://www.beta.inegi.org.mx/proyectos/ccpv/2000/

INSTITUTO NACIONAL DE ESTADÍSTICA Y GEOGRAFÍA (2010). XII Censos General de Población y Vivienda 2010. [En línea] http://www.beta.inegi.org.mx/proyectos/ccpv/2010/default.html

INSTITUTO NACIONAL DE ESTADÍSTICA Y GEOGRAFÍA (2014). Censos Económicos 2009. México. [En línea] http://internet.contenidos.inegi.org.mx/contenidos/productos//prod_serv/contenidos/espanol/bvinegi/productos/nueva_estruc/702825077952.pdf

INSTITUTO NACIONAL DE ESTADÍSTICA Y GEOGRAFÍA (2015). Encuesta intercensal 2015, México. [En línea] http://www.beta.inegi.org.mx/proyectos/enchogares/especiales/intercensal/ JARQUÍN SÁNCHEZ, N. H., CASTELLANOS SUÁREZ, J. A. y SANGERMAN-JARQUÍN, D. A. (2017). «Pluriactividad y agricultura familiar: retos del desarrollo rural en México». Revista Mexicana de Ciencias Agricolas, 8(4), 949-963. 
LOZADA, M., ROJAS, I., MASTRETTA, A., PONCE-MENDOZA, A., BURGEFF, C., ORJUELA, A. y OLIVEROS, O. (2017). «Las milpas de México». OIKOS, 17.

MARTÍNEZ BORREGO, E. (1991). Organización de productores y movimiento campesino. México: Siglo XXI.

POLE, K. (2009). «Diseño de metodologías mixtas. Una revisión de las estrategias para combinar metodologías cuantitativas y cualitativas». Revista Renglones, 60, 37-42.

SALAS, H. «Apuntes para comprender la transformación de los territorios rurales en comunidades ejidales de Nativitas al sur de Tlaxcala». En SALAS, H. y PALETA, G. (eds.). (2016) Las transformaciones rurales en la globalización: trabajo, cambios territoriales y ruralidades. Ciudad de México: Universidad Nacional Autónoma de México, Instituto de Investigaciones Antropológicas, pp. 151-170.

ROJAS, T. y GUTIERRÉZ, I. (2016). Letras y huellas del maí: del siglo XVI a 1914. Ciudad de México: Consejo Nacional de Ciencia y Tecnología, Red de Etnoecología y Patrimonio Biocultural, Juan Pablos Editor.

TORRES SALCIDO, G. (2009). De la producción de maí al consumo social de la tortilla. Políticas de producción y abastecimiento urbano. Ciudad de México: UNAM, Coordinación de Humanidades, Centro de Investigaciones Interdisciplinarias en Ciencias y Humanidades.

WARMAN, A. (1988). La historia de un bastardo: Maíry capitalismo. México: Fondo de Cultura Económica. 